Research Article

\title{
Prognostic Value of ctDNA Mutation in Melanoma: A Meta-Analysis
}

\author{
Yang Zheng, ${ }^{1}$ Hongyan Sun, ${ }^{2}$ Lele Cong, ${ }^{1}$ Chenlu Liu, ${ }^{2}$ Qian Sun, ${ }^{1}$ Nan Wu, \\ and Xianling Cong ${ }^{1}$
}

${ }^{1}$ Department of Dermatology, China-Japan Union Hospital of Jilin University, Changchun, China
${ }^{2}$ Biobank, China-Japan Union Hospital of Jilin University, Changchun, China

Correspondence should be addressed to Xianling Cong; congxl@jlu.edu.cn

Received 29 October 2020; Revised 18 March 2021; Accepted 26 March 2021; Published 5 May 2021

Academic Editor: Prasanna Kumar Santhekadur

Copyright (C) 2021 Yang Zheng et al. This is an open access article distributed under the Creative Commons Attribution License, which permits unrestricted use, distribution, and reproduction in any medium, provided the original work is properly cited.

Purpose. Melanoma is the most aggressive form of skin cancer. Circulating tumor DNA (ctDNA) is a diagnostic and prognostic marker of melanoma. However, whether ctDNA mutations can independently predict survival remains controversial. This metaanalysis assessed the prognostic value of the presence or change in ctDNA mutations in melanoma patients. Methods. We identified studies from the PubMed, EMBASE, Web of Science, and Cochrane databases. We estimated the combined hazard ratios (HRs) for overall survival (OS) and progression-free survival (PFS) using either fixed-effect or random-effect models based on heterogeneity. Results. Sixteen studies including 1,781 patients were included. Both baseline and posttreatment detectable ctDNA were associated with poor OS (baseline detectable vs. undetectable, pooled $\mathrm{HR}=1.97,95 \% \mathrm{CI}=1.64-2.36, P<0.00001$; baseline undetectable vs. detectable, pooled $\mathrm{HR}=0.19,95 \% \mathrm{CI}=0.11-0.36, P<0.00001$; posttreatment detectable vs. undetectable, pooled $\mathrm{HR}=2.36,95 \% \mathrm{CI}=1.30-4.28, P=0.005)$. For PFS, baseline detectable ctDNA may be associated with adverse PFS (baseline detectable vs. undetectable, pooled $\mathrm{HR}=1.41,95 \% \mathrm{CI}=0.84-2.37, P=0.19$; baseline undetectable vs. detectable, pooled $\mathrm{HR}=0.43,95 \% \mathrm{CI}=0.19-0.95, P=0.04)$ and baseline high ctDNA and increased ctDNA were significantly associated with adverse PFS (baseline high vs. low/undetectable, pooled $\mathrm{HR}=3.29,95 \% \mathrm{CI}=1.73-6.25, P=0.0003$; increase vs. decrease, pooled $\mathrm{HR}=4.48,95 \% \mathrm{CI}=2.45-8.17, P<0.00001)$. The baseline $\mathrm{BRAF}^{\mathrm{V} 600} \mathrm{ctDNA}$ mutation-positive group was significantly associated with adverse OS compared with the baseline ctDNA-negative group (pooled HR $=1.90,95 \% \mathrm{CI}=1.58-2.29, P<0.00001$ ). There were no significant differences in PFS between the baseline BRAF ${ }^{\vee 00} \mathrm{ctDNA}$ mutation-detectable group and the undetectable group (pooled $\mathrm{HR}=1.02,95 \% \mathrm{CI}=0.72-1.44, P=0.92$ ). Conclusion. The presence or elevation of $\mathrm{ctDNA}^{\mathrm{m}} \mathrm{\text {mation }}$ or $\mathrm{BRAF}^{\mathrm{V} 600}$ ctDNA mutation was significantly associated with worse prognosis in melanoma patients.

\section{Introduction}

Melanoma is the most aggressive form of skin cancer, originating from pigment-producing melanocytes. Though it accounts for only $10 \%$ of all skin cancers, it is responsible for more than $80 \%$ of skin cancer-related deaths. The development of targeted therapy (MAPK pathway inhibitors) $[1,2]$ and immunotherapy (checkpoint inhibitors) [3-5] has led to substantial improvements in overall survival (OS). However, a significant number of patients develop acquired resistance or do not benefit from therapy and, in some cases, therapy can be toxic $[6,7]$. Therefore, the monitoring of disease progression and prognosis of patients are of vital importance and contribute to improving the quality of life of melanoma patients.

In recent years, many studies have focused on the value of circulating tumor DNA (ctDNA) in monitoring disease status and prognosis [8-12]. Some studies have focused on the prognostic value of ctDNA in melanoma patients, although the results are controversial. A study [13] conducted by Tan et al. confirmed that ctDNA detection before surgery and postoperatively can identify melanoma patients at highest risk of relapse, although there was no significant association between detectable ctDNA (at baseline or 
postoperatively) and OS. Lee et al. [14] proposed that OS was significantly worse for patients with detectable ctDNA postsurgery and high-risk stage II/III melanoma. In the study [15] by Seremet et al., undetectable ctDNA at baseline remained significantly correlated with progression-free survival (PFS) and OS in metastatic melanoma patients treated with anti-PD1 therapy. Forthun et al. conducted a study [16] of bevacizumab in the treatment of metastatic malignant melanoma and showed that $\leq 1 \%$ BRAF/NRASpositive ctDNA before and during treatment reflected a positive response to therapy, through an increase in the durations of PFS and OS. A study by Board et al. [17] indicated that cfDNA BRAF detection is not associated with poorer PFS in stage III/IV advanced melanoma. Besides, there is still a lack of systematic evidence to prove the prognostic value of ctDNA in melanoma patients. Sequencing data identified melanoma as the most frequently mutated tumor type analyzed by The Cancer Genome Atlas (TCGA) [18]. Therefore, the aim of this meta-analysis was to evaluate the prognostic significance of ctDNA mutations in melanoma patients, in terms of OS and PFS.

\section{Methods}

2.1. Data Sources and Searches. We conducted systematic searches of the PubMed, EMBASE, Web of Science, and Cochrane databases for entries up until March, 2020 without language or study period restrictions. The search terms included ("ctDNA" or "circulating tumor DNA" or "circulating DNA" or "free DNA" or "cell free DNA" or "plasma DNA" or "serum DNA") and ("Melanoma" or "Skin cancer" or "Skin neoplasia" or "skin neoplasm") and ("prediction" or "outcome" or "predictive" or "prognosis" or "prognostic"). A manual search of related articles and references cited in these articles was performed to identify all available studies.

2.2. Inclusion and Exclusion Criteria. Studies were included in the analysis if they met the following criteria: (1) all patients enrolled in the study were diagnosed with melanoma, (2) ctDNA mutation was assessed using plasma or serum, (3) endpoints included PFS or OS, and sufficient data were presented for determining or calculating the hazard ratio (HR) and 95\% confidence interval (95\% CI).

Studies were excluded if (1) fewer than 15 patients were included in the statistical analysis of prognosis value of ctDNA, (2) samples were not drawn from peripheral blood (e.g., they came from cerebrospinal fluid or lymphatic fluid), and (3) nonresearch publications such as editorials, reviews, and letters, or the publications were duplicated articles.

2.3. Data Extraction and Quality Assessment. Two investigators independently reviewed all eligible studies and extracted the following information: first author name, publication year, country, number of patients analyzed, tumor stage, sample origin, time of sample collection, clinical therapy, method/platform of ctDNA detection, target genes/variants, cutoff value, number of experimental/ control samples, positive ratio, and endpoint, and follow-up duration and confounding factors, if provided. When the article had multiple queues or groups of patients, and an HR for survival curve was provided for each queues/groups, results of all these queues/groups were recorded as independent data. The quality of all included studies was evaluated using the Newcastle-Ottawa Quality Assessment Scale (NOS) [19]. An NOS score of 5-9 stars was considered indicative of a high quality meta-analysis.

2.4. Statistical Analysis. Hazard ratios (HRs) and their $95 \%$ CIs for PFS and OS were recorded to clarify the prognostic value of ctDNA. For studies in which HRs and 95\% CIs were not available, we extracted survival rates from Kaplan-Meier curves by using Engauge Digitizer version 4.1 [20]. To assess the heterogeneity among studies, pooled HRs were initially calculated using a fixed effects model. If there was significant heterogeneity among studies $\left(I^{2}>50 \%\right)$, the random effects model was adopted [21]. $I^{2}>50 \%$ and $P<0.05$ were considered significant for heterogeneity.

For forest plots with more than 10 included studies or results, we evaluated publication bias using funnel plots for visual inspection and conducted quantitative estimations using Egger's test. Sensitivity analysis was performed by excluding each study in turn to assess the stability of the results. All analyses were carried out using Review Manager version 5.3 (The Cochrane Collaboration, Copenhagen: The Nordic Cochrane Center, 2012) and STATA version 12.0 (STATA Corporation, College Station, TX, USA).

\section{Results}

3.1. Search Results. A total of 2,365 articles were identified after removing duplicates. By reviewing titles and abstracts, 2,305 articles were excluded, of which 2,114 were not related to the disease or subject of our meta-analysis, 86 were reviews or systematic evaluations, and 105 were abstracts, conference papers, or case reports. After detailed reading and evaluation of 60 articles, we excluded eight articles because the number of patients analyzed for prognosis was less than 15. Twelve articles were excluded for lack of prognostic information. Twenty-one articles were excluded because they did not provide sufficient data to extract HRs for PFS or OS. Further three articles with or suspected to have overlapping study populations were excluded. Finally, 16 articles [12-17, 22-31] proved eligible for inclusion and were analyzed (Figure 1).

3.2. Literature Characteristics and Quality. The characteristics and quality of studies included in the meta-analysis are described in Table 1 . The 16 studies [12-17, 22-31] were published between 2007 and 2020, and the sample size of ctDNA prognosis analyses ranged from 20 to 551, with an overall total of 1,781. Among the studies, four [12, 13, 25, 29] were from Australia, and one study each was from the UK [14], Poland [22], Spain [28], Norway [16], Italy [24], Belgium [15], France [27], and USA [31]. In addition, one study [23] was from Belgium and Germany. The samples from one study [17] were obtained from the participants of a phase 2 


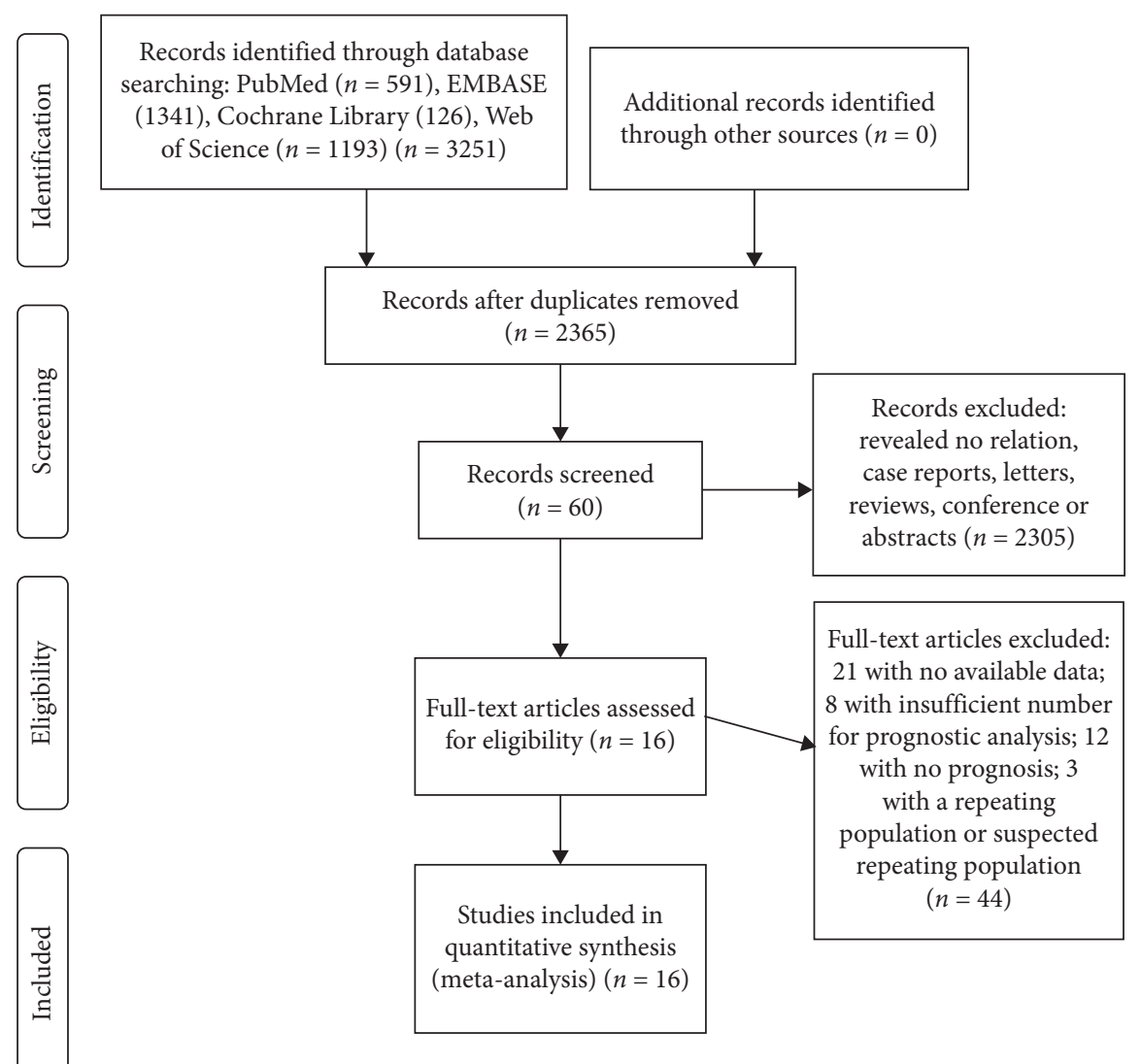

Figure 1: Study selection strategy and flow diagram.

clinical trial in which the patients were from 10 countries. The samples from one study [26] were obtained from the participants of a phase 3 clinical trial in which the patients were from 12 countries. One study [30] included samples from the participants of four clinical trials, each involving patients from more than one country. Since two of the clinical trials enrolled the same patient populations, we chose the one with larger sample size for meta-analysis. In addition to the study [30] from three clinical trials, another three studies $[12,23,26]$ were grouped by cohort or drug therapy. Therefore, we considered them as independent studies. All the patients in 15 studies had advanced melanoma, while the patients in one study [14] were at stage IIIII. Plasma ctDNA levels were assessed in 12 studies [12-16, 22, 23, 25-27, 29, 30], serum ctDNA levels were assessed in three studies [17, 24, 31], and ctDNA was extracted from serum and plasma samples in one study [28]. Twelve studies [12, 13, 15, 16, 22-24, 26-29, 31] analyzed ctDNA from blood samples before and after treatment, and four studies $[14,17,25,30]$ analyzed ctDNA before treatment. However, among these 12 studies $[12,13,15,16,22-24,26-29,31]$, PFS or OS analysis was not performed in eight studies [12, 15, 22, 24, 26-29] using posttreatment data, and pretreatment data was not performed in one study [31]. Droplet digital PCR (ddPCR) was used to detect ctDNA in blood samples in 11 studies $[12-16,22-26,29]$. Only the BRAF ${ }^{\mathrm{V} 600} \mathrm{ctDNA}$ mutation was detected in seven studies $[17,22,24,26,28,30,31]$, and multiple genes were detected in another study [23], although PFS or OS analysis of the $\mathrm{BRAF}^{\mathrm{V} 600}$ mutation was available. The other studies $[12-16,25,27,29]$ all detected the mutations in multiple genes. The quality of studies included was evaluated by NOS, and all the studies received at least six stars, all of which were regarded as high quality.

\subsection{Prognostic Value of ctDNA in Melanoma}

3.3.1. Meta-Analysis of ctDNA Predicting OS. A total of 11 studies $[13,15,16,22-24,26-30]$ assessed the relationship between baseline ctDNA and OS. Seven studies (10 results) $[13,22-24,26,28,30]$ analyzed the effect of baseline detectable ctDNA on OS compared with baseline undetectable ctDNA. The multivariate results of one study [22] analyzing the effect of the log-transformed concentration of baseline ctDNA on prognosis were not included in the analysis. As shown in Figure 2(a), the risk was significantly higher in the baseline ctDNA-positive group than in the baseline ctDNAnegative group in terms of mortality (pooled $\mathrm{HR}=1.97,95 \%$ $\mathrm{CI}=1.64-2.36, P<0.00001)$. The $I^{2}$ statistical heterogeneity was not significant $\left(I^{2}=0 \%\right)$. This suggests that baseline detectable ctDNA is associated with adverse OS.

Three studies $[15,27,29]$ indicated the association of baseline undetectable ctDNA with OS compared with baseline detectable ctDNA. Our results showed that, in melanoma, the risk of mortality in the baseline undetectable 


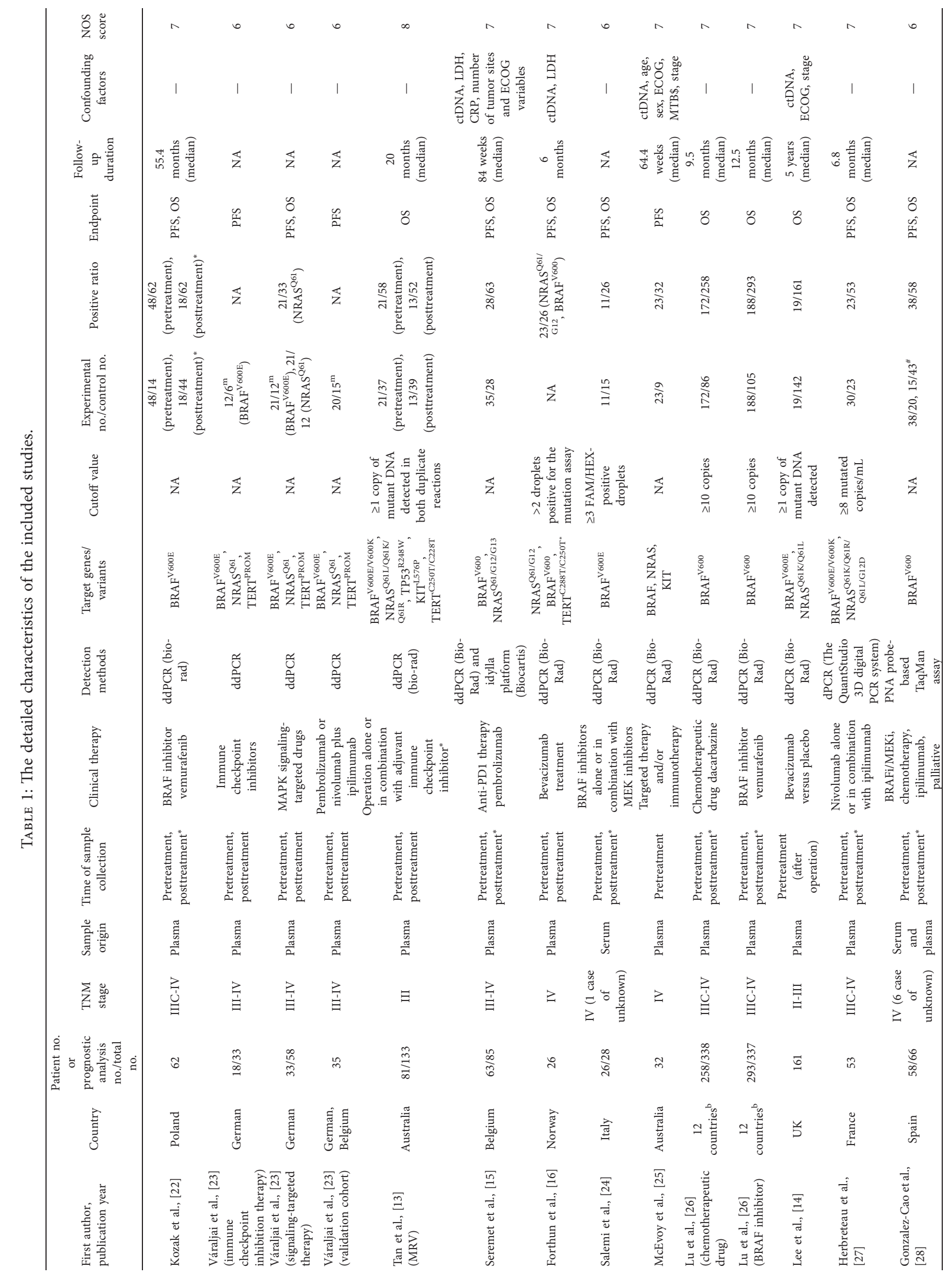




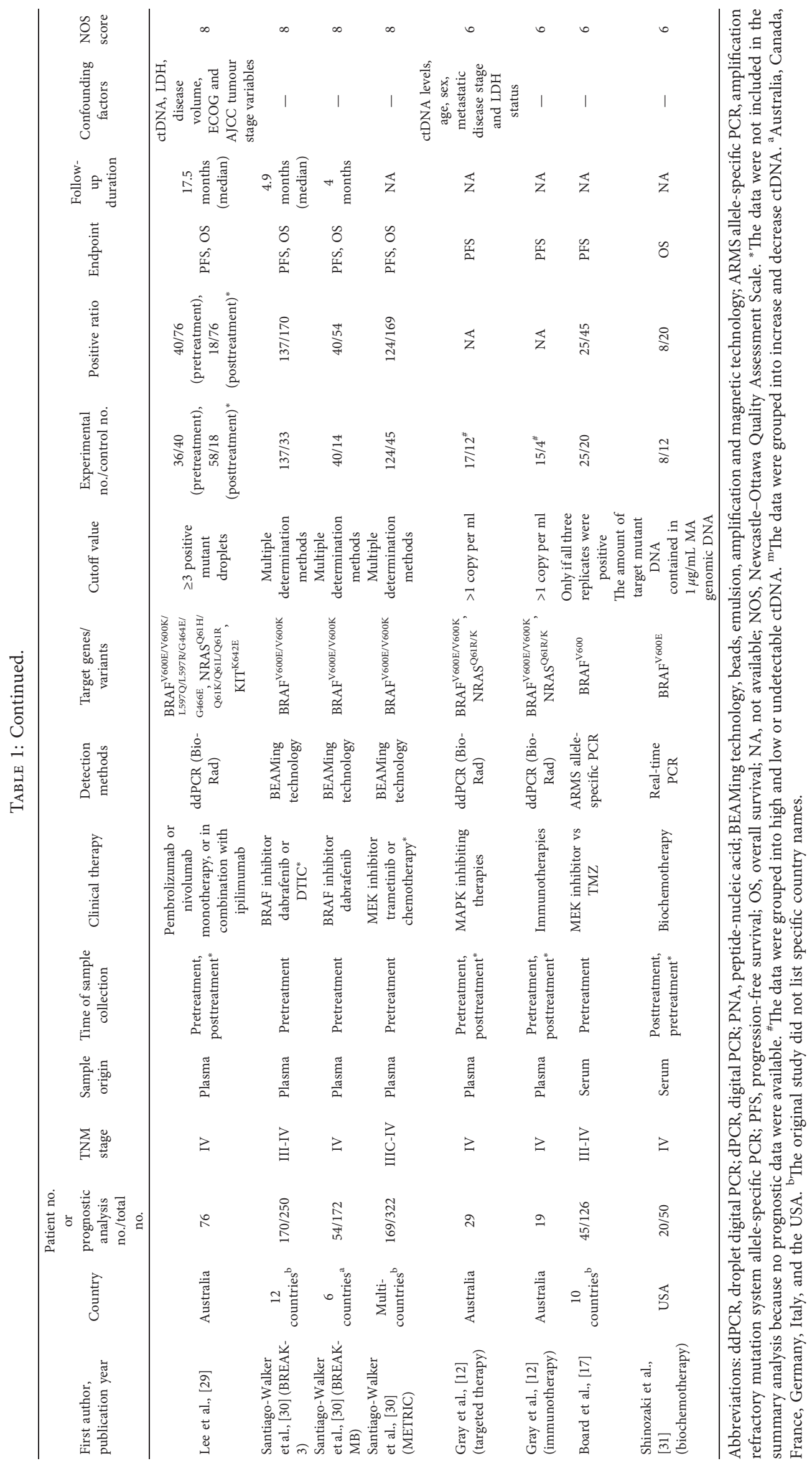




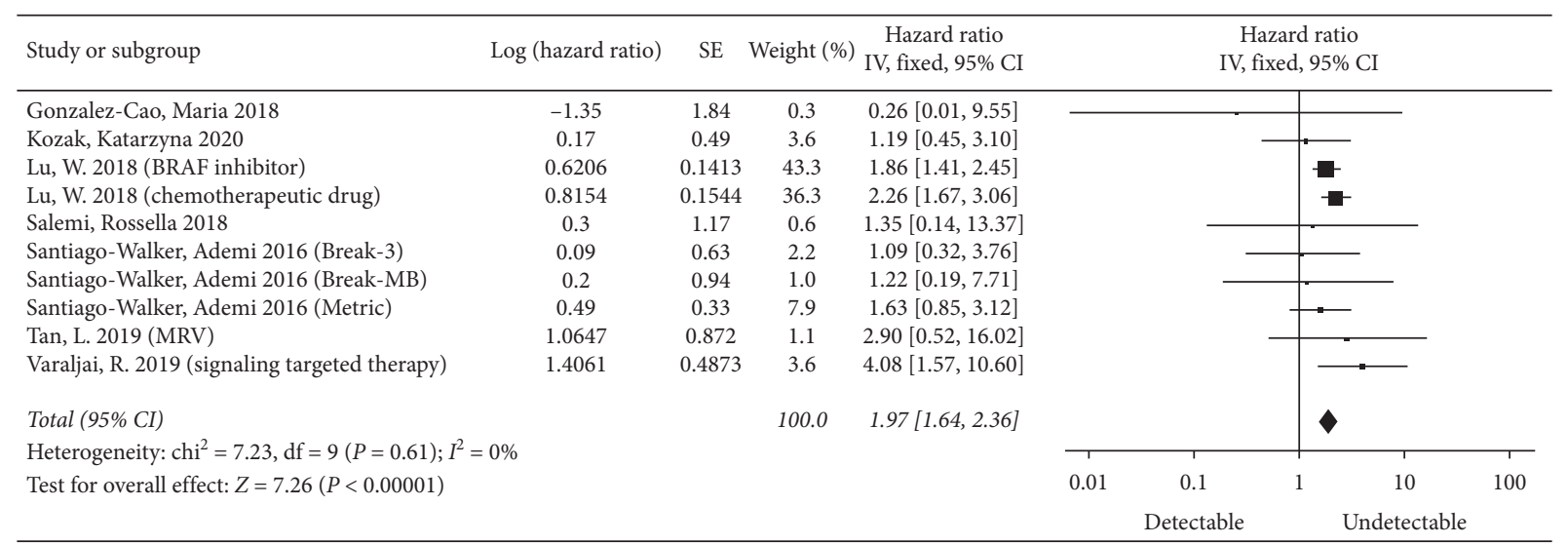

(a)

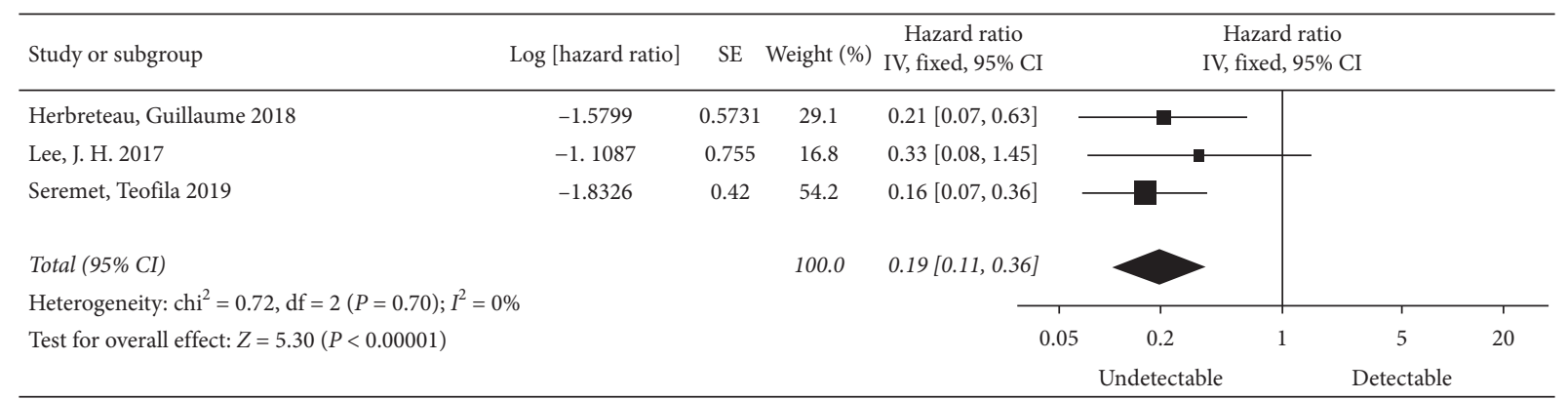

(b)

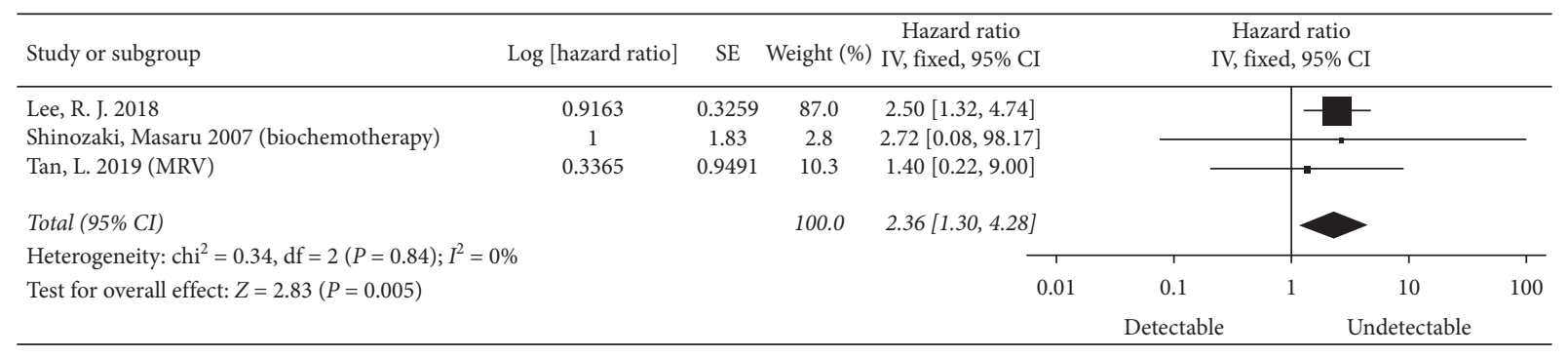

(c)

FIgUre 2: Meta-analysis of ctDNA predicting OS. (a) Effect of baseline ctDNA on OS in melanoma (detectable vs. undetectable). (b) Effect of baseline ctDNA on OS in melanoma (undetectable vs. detectable). (c) Effect of posttreatment ctDNA on OS in melanoma (detectable vs. undetectable).

ctDNA group was significantly lower than that in the baseline detectable ctDNA group (pooled $\mathrm{HR}=0.19,95 \%$ $\mathrm{CI}=0.11-0.36, P<0.00001)$, with no heterogeneity $\left(I^{2}=0 \%\right)$ (Figure 2(b)). This suggests that baseline undetectable ctDNA is associated with better OS.

In addition, one study [16] analyzed the relationship between baseline high ctDNA and OS compared with baseline undetectable or low ctDNA, suggesting that baseline high ctDNA was associated with adverse OS. One study [28] suggested that baseline undetectable or low ctDNA was significantly associated with better OS compared with baseline high ctDNA. One study [26] also showed a relationship between high versus low, high versus undetectable, and low versus undetectable and OS, with statistically significant differences. Another study [29] analyzed patients with posttreatment negative-ctDNA and baseline undetectable ctDNA was significantly associated with better OS compared with baseline detectable ctDNA. The above results were not included in the analysis due to different classification methods. These results suggest that baseline detectable ctDNA is associated with adverse OS.

Four studies [13, 14, 16, 31] evaluated the relationship between posttreatment ctDNA and OS, although one [16] was not included in the analysis because of the different grouping method. Our results showed that in melanoma, compared with the posttreatment undetectable ctDNA group, the posttreatment detectable ctDNA group was associated with adverse OS (pooled $\mathrm{HR}=2.36,95 \%$ $\mathrm{CI}=1.30-4.28, P=0.005)$, with no heterogeneity $\left(I^{2}=0 \%\right)$ (Figure 2(c)). One study [16] that was not included in the analysis examined the relationship between posttreatment high ctDNA and OS compared with posttreatment undetectable and low ctDNA, suggesting that posttreatment high ctDNA was significantly associated with adverse OS. These 
results suggest that posttreatment detectable ctDNA is associated with adverse OS.

3.3.2. Meta-Analysis of ctDNA Predicting PFS. A total of 12 studies $[12,15-17,22-25,27-30]$ assessed the relationship between baseline ctDNA and PFS. Seven studies (nine results) $[17,22-25,28,30]$ analyzed the effect of baseline detectable ctDNA on PFS compared with baseline undetectable ctDNA. The multivariate results of one study [22] analyzing the effect of the log-transformed concentration of baseline ctDNA on prognosis were not included in the analysis. As shown in Figure 3(a), the baseline detectable ctDNA group benefited less than the baseline undetectable ctDNA group in terms of treatment response, although the differences were not statistically significant (pooled $\mathrm{HR}=1.41,95 \% \mathrm{CI}=0.84-2.37, P=0.19)$. The $I^{2}$ statistical heterogeneity was high $\left(I^{2}=55 \%\right)$; thus, a random-effects model was used.

Three studies $[15,27,29]$ indicated the association of baseline undetectable ctDNA with PFS compared with baseline detectable ctDNA. The results showed that baseline undetectable ctDNA was significantly associated with better PFS (pooled $\mathrm{HR}=0.43,95 \% \mathrm{CI}=0.19-0.95, \quad P=0.04$, Figure $3(\mathrm{~b})$ ). The $I^{2}$ statistical heterogeneity was high $\left(I^{2}=73 \%\right)$; thus, a random-effects model was used.

Two studies (three results) [12,16] analyzed the differences between the baseline high ctDNA group and the baseline undetectable or low ctDNA group and evaluated PFS. The baseline high ctDNA group benefited less than the baseline undetectable or low ctDNA group in terms of treatment response (pooled $\mathrm{HR}=3.29,95 \% \mathrm{CI}=1.73-6.25$, $P=0.0003)$, with no heterogeneity $\left(I^{2}=0 \%\right)$ (Figure $3(\mathrm{c})$ ).

In addition, data provided in two studies $[28,29]$ not included in the above analysis due to different grouping methods showed that baseline undetectable or low ctDNA was significantly associated with better PFS compared with baseline high ctDNA [28] and that in patients with posttreatment negative-ctDNA, baseline undetectable ctDNA was significantly associated with better PFS compared with baseline detectable ctDNA [29]. These results suggest that baseline detectable ctDNA may be associated with adverse PFS.

The relationship between posttreatment ctDNA detection and PFS was analyzed in one study [16], which suggested that posttreatment high ctDNA was significantly associated with adverse PFS compared with posttreatment undetectable or low ctDNA. Due to the insufficient number of studies, no analysis was carried out.

One study (three results) [23] indicated that the association of ctDNA changed with PFS. The increased ctDNA group had less therapeutic benefit than the decreased ctDNA group (pooled $\mathrm{HR}=4.48,95 \% \mathrm{CI}=2.45-8.17, P<0.00001$ ), with no heterogeneity $\left(I^{2}=0 \%\right)$ (Figure $3(\mathrm{~d})$ ).

3.4. Prognostic Value of the ctDNA BRAF ${ }^{V 600}$ Mutation in Melanoma. We only further analyzed the effects of baseline $\mathrm{BRAF}^{\mathrm{V} 600}$ mutation detectable ctDNA on PFS and OS, compared with baseline $\mathrm{BRAF}^{\mathrm{V} 600}$ mutation undetectable ctDNA because of different grouping methods and the limited number of studies. Our results showed that the risk was significantly higher in the baseline $\mathrm{BRAF}^{\mathrm{V} 600}$ ctDNA mutation-positive group than in the baseline ctDNA-negative group in terms of mortality (pooled $\mathrm{HR}=1.90,95 \% \mathrm{CI}=1.58-2.29, P<0.00001)$, with no heterogeneity $\left(I^{2}=0 \%\right)$ (Figure $\left.4(\mathrm{a})\right)$. The differences between the baseline BRAF ${ }^{\mathrm{V} 600}$ ctDNA mutation detectable group and undetectable group were not statistically significant in terms of treatment response (pooled $\mathrm{HR}=1.02$, 95\% $\mathrm{CI}=0.72-1.44, P=0.92)$, with low heterogeneity $\left(I^{2}=4 \%\right)$ (Figure 4(b)).

3.5. Heterogeneity Analysis. A subgroup analysis was performed on the forest plot with the largest number of studies to identify the factors that may influence interstudy heterogeneity, including the effects of baseline detectable ctDNA on PFS and OS, compared with baseline undetectable ctDNA.

Table S1 shows the results of subgroup analyses, stratified by target gene, sample origin, method, and data source. There was no heterogeneity among the subgroups that assessed OS $\left(I^{2}=0 \%\right)$. In most subgroups, baseline detectable ctDNA was significantly associated with adverse OS, although there was no significant correlation between baseline detectable ctDNA and OS in the non-ddPCR ctDNA evaluation subgroup and in the subgroup whose data were extracted from the survival curve (pooled $\mathrm{HR}=1.41,95 \%$ $\mathrm{CI}=0.82-2.43, \quad P=0.21 ; \quad$ pooled $\quad \mathrm{HR}=1.36, \quad 95 \%$ $\mathrm{CI}=0.85-2.15, P=0.20$, respectively). For the subgroups that evaluated PFS, heterogeneity was significantly reduced according to whether the target gene included only $\mathrm{BRAF}^{\mathrm{V} 600}$ or not, and according to whether the data was directly provided by the studies or extracted from the survival curve. As it happened, the two subgroups included the same studies, whose target genes were $\mathrm{BRAF}^{\mathrm{V} 600}$, and data were extracted from the survival curve, which showed no significant correlation between baseline detectable ctDNA and PFS (pooled $\mathrm{HR}=1.02,95 \% \mathrm{CI}=0.72-1.44, P=0.92$, $I^{2}=4 \%$ ). Meanwhile, the subgroup whose data was provided directly by the studies suggested that baseline detectable ctDNA was associated with adverse PFS (pooled $\mathrm{HR}=4.02$, $\left.95 \% \mathrm{CI}=1.88-8.59, \quad P=0.0003, I^{2}=1 \%\right)$. These results suggest that the sources of heterogeneity may be the method of ctDNA assessment, target genes, and data sources.

3.6. Sensitivity Analysis. Sensitivity analysis based on the change in the combined effect size by excluding the included studies one by one is an important method mainly used to evaluate the robustness and reliability of the combined results of meta-analysis. After sensitivity analysis, the overall effect sizes of the two meta-analyses were significantly modified. Among them, one study [14] had a significant impact on the effect of posttreatment detectable ctDNA on OS. After excluding this study, the overall HR changed from $2.36(95 \% \quad \mathrm{CI}=1.30-4.28, \quad P=0.005) \quad$ to $1.61 \quad(95 \%$ $\mathrm{CI}=0.31-8.40, P=0.57)$, and the heterogeneity remained at 0 . Two studies $[15,27]$ had a significant impact on the effect of baseline undetectable ctDNA on PFS. After excluding one 


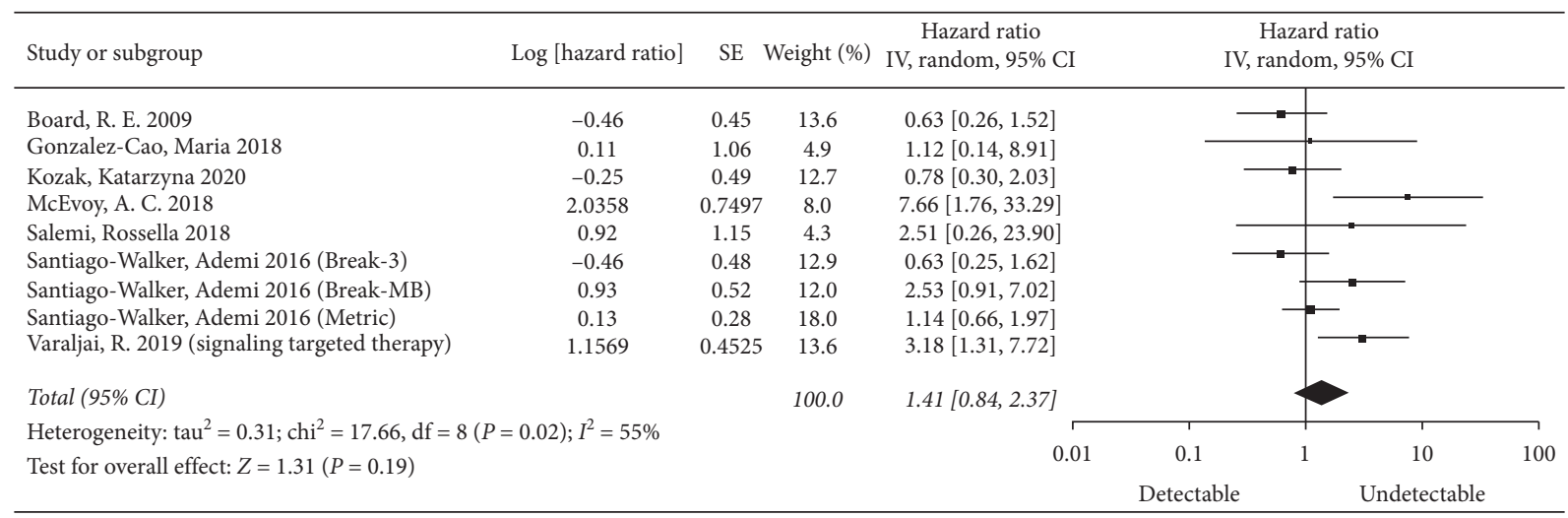

(a)

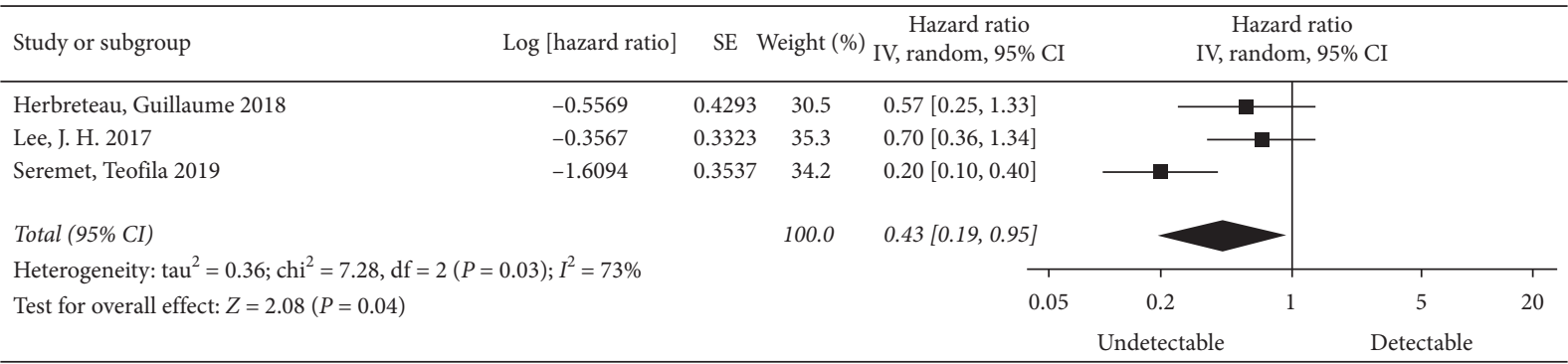

(b)

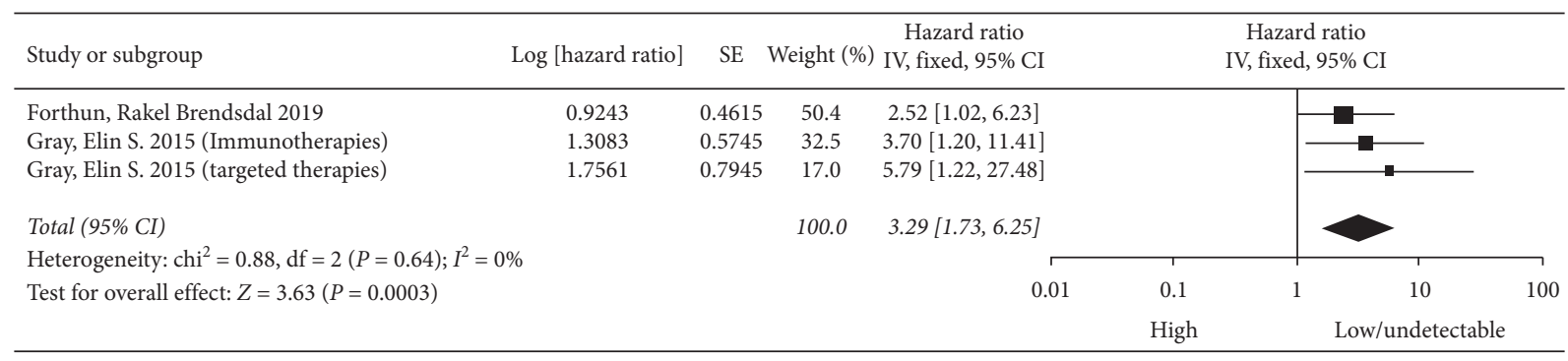

(c)

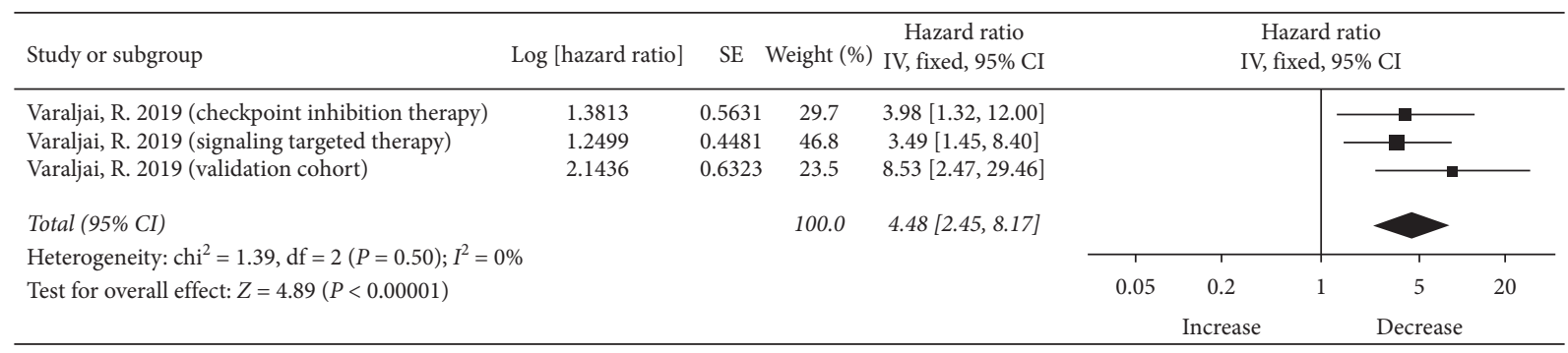

(d)

Figure 3: Meta-analysis of ctDNA predicting PFS. (a) Effect of baseline ctDNA on PFS in melanoma (detectable vs. undetectable). (b) Effect of baseline ctDNA on PFS in melanoma (undetectable vs. detectable). (c) Effect of baseline ctDNA on PFS in melanoma (high vs. low/undetectable). (d) Effect of ctDNA change on PFS in melanoma (increase vs. decrease).

study [27], the overall HR changed from $0.43 \quad(95 \%$ $\mathrm{CI}=0.19-0.95, \quad P=0.04)$ to $0.38(95 \% \mathrm{CI}=0.11-1.28$, $P=0.12)$ and the heterogeneity was almost unchanged (from $I^{2}=73 \%$ to $I^{2}=85 \%$ ). After excluding the other study [15], the overall HR changed to 0.65 (95\% CI $=0.39-1.09$, $P=0.10)$ and the heterogeneity decreased to 0 .
3.7. Publication Bias. Funnel plots and Egger's test were used to assess publication bias for forest plots containing more than 10 studies or results, thus assessing the impact of baseline detectable ctDNA on OS. The results revealed no evidence of significant publication bias (Egger's test, $P=0.306)$ (Figure 5). 


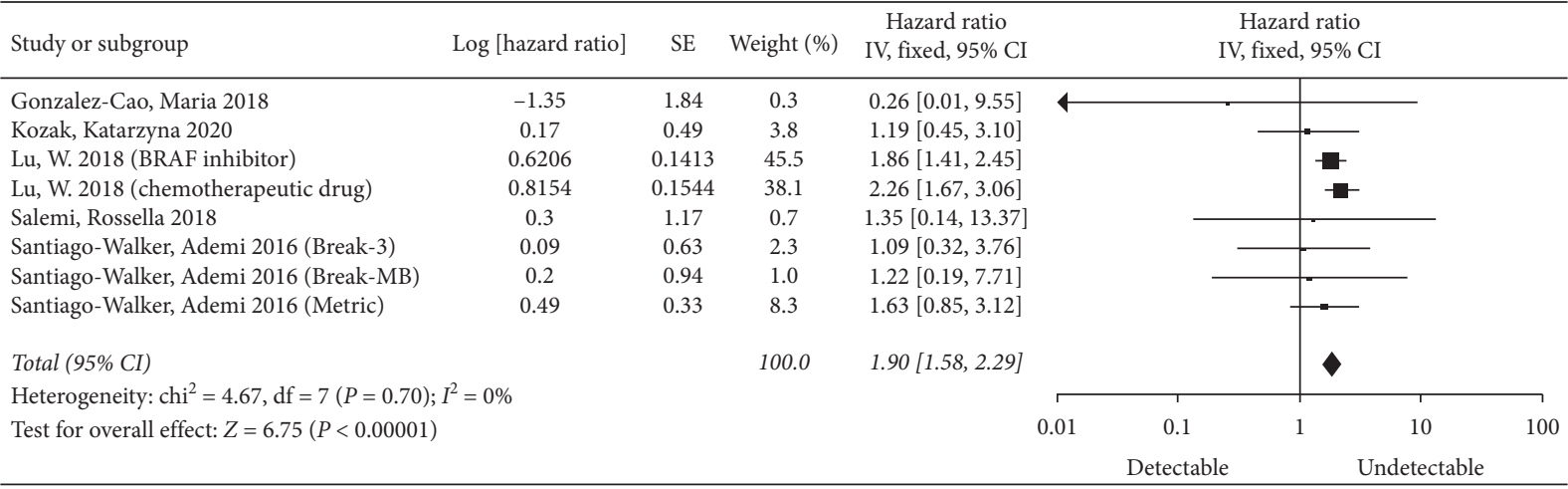

(a)

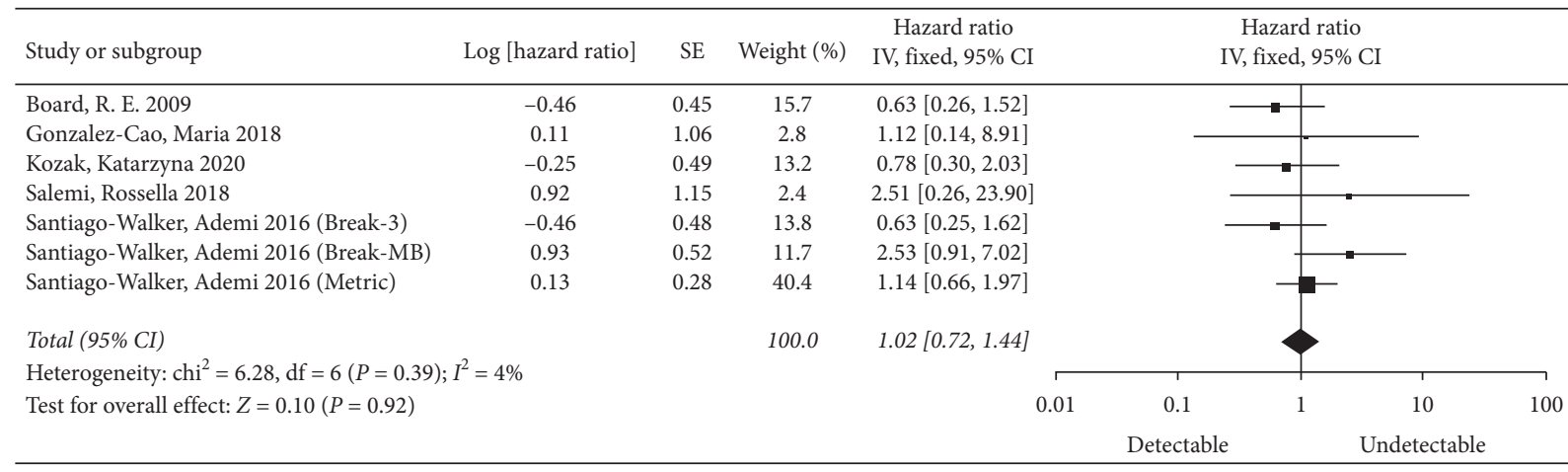

(b)

FIgURE 4: Meta-analysis of the BRAF ${ }^{\mathrm{V} 600} \mathrm{ctDNA}$ mutation predicting OS or PFS. (a) Effect of baseline BRAF ${ }^{\mathrm{V} 600} \mathrm{ctDNA} \mathrm{mutation}$ on OS in melanoma (detectable vs. undetectable). (b) Effect of baseline BRAF ${ }^{\mathrm{V} 600} \mathrm{ctDNA}$ mutation on PFS in melanoma (detectable vs. undetectable).

\section{Discussion}

In 2012, melanoma was the 15th most common cancers worldwide [32]. Unfortunately, the worldwide incidence of cutaneous melanoma has been increasing at a faster rate each year than that of any other type of cancer [33]. The treatment of melanoma has been revolutionized in the past decade. In particular, the PFS and OS in melanoma patients have improved significantly with the introduction of immune checkpoint inhibitors and the new selective tyrosine kinase inhibitors, including the BRAF and MEK inhibitors [34], although there are still a certain proportion of patients experiencing early tumor recurrence or progression. As a result, major efforts have been made to better monitor the evolution of the disease and the prognosis of patients, such as considering histopathological features, patient characteristics, biochemical indicators, and genetic mutations [33]. Sequencing data has shown that melanoma has a median mutation rate of 10 mutations/Mb, the highest of all cancers so far analyzed by the TCGA network [18]. Meta-analysis has demonstrated that BRAF mutation is an absolute risk factor for survival in melanoma patients [35]. However, it is difficult to study genomic changes in repeated tumor biopsies during treatment. Since Mandel and Metais [36] first discovered circulating nucleic acids in the blood of healthy humans in 1948, ctDNA has been widely used in many disciplines. Analysis of ctDNA can provide a noninvasive method to assess prognosis and response to treatment [12].
Our meta-analysis assessed the prognostic significance of ctDNA mutation in baseline or treatment samples from melanoma patients and played a guiding role in the clinical treatment of melanoma.

This meta-analysis included 16 studies including 1,781 melanoma patients for a prognostic analysis. We analyzed differences in OS and PFS at the endpoint of observation for mutations in ctDNA at baseline and after treatment. We also calculated the relevant HRs and 95\% CIs. The results revealed that ctDNA mutation was significantly associated with the prognosis of melanoma patients. Specifically, patients with detectable ctDNA mutation tended to have adverse OS compared to patients where ctDNA mutation was not detected, either at baseline or after treatment. Patients with low or undetectable ctDNA mutation at baseline tend to have better PFS compared with patients with high ctDNA mutation, and baseline detectable ctDNA may be associated with adverse PFS. Patients with decreased ctDNA levels tend to have favorable PFS compared with patients with increased ctDNA levels. CtDNA BRAF ${ }^{\mathrm{V} 600}$ mutation is also a prognostic biomarker with similar prognostic value. Patients with detectable $\mathrm{BRAF}^{\mathrm{V} 600} \mathrm{ctDNA}$ at baseline tend to have worse OS compared to patients with undetectable $\mathrm{BRAF}^{\mathrm{V} 600} \mathrm{ctDNA}$, while baseline detectable $\mathrm{BRAF}^{\mathrm{V} 600} \mathrm{ctDNA}$ may be associated with worse PFS.

The result of our subgroup analysis stratified by target gene showed that $\mathrm{BRAF}^{\mathrm{V} 600}$ mutation was significantly associated with worse OS, while patients with BRAF B $600^{2}$ ctDNA tended to have poor PFS, although without statistical 


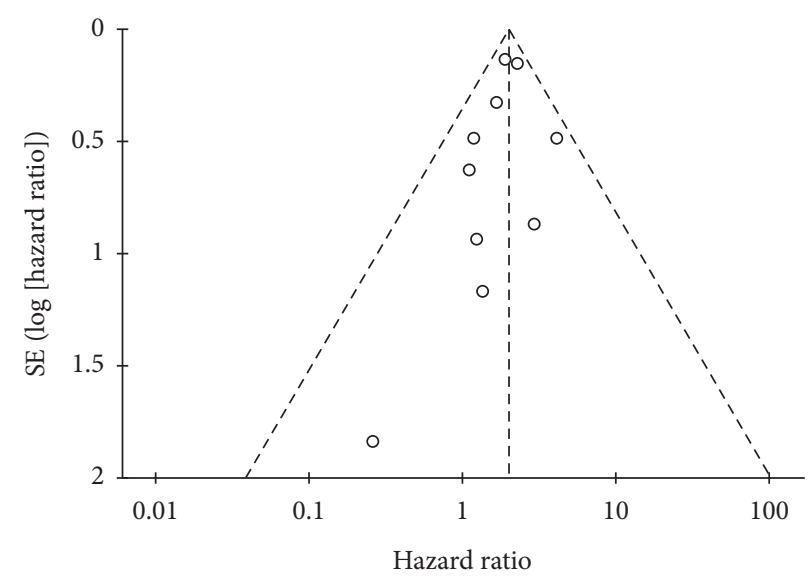

(a)

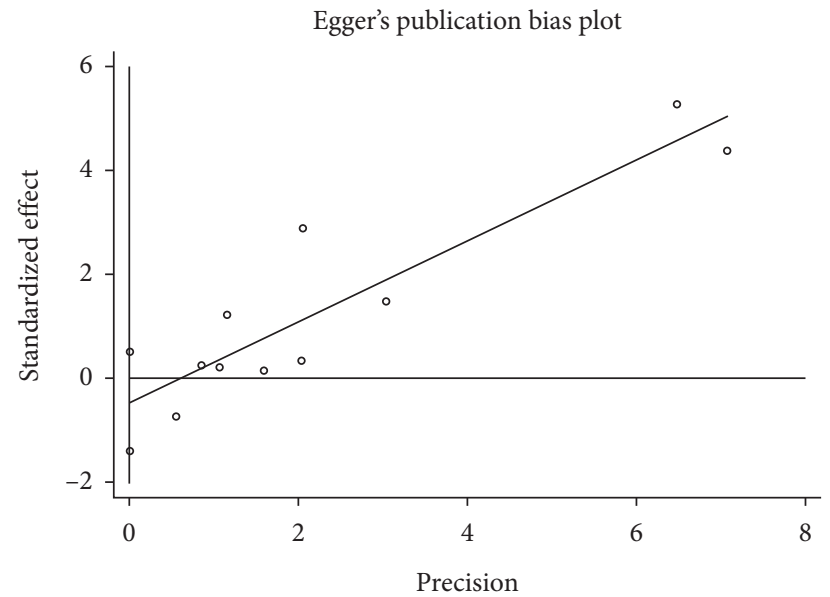

(b)

Figure 5: Funnel plot and Egger's test of meta-analysis for the association between baseline ctDNA and OS.

significance. However, the subgroups in which the association between multiple gene ctDNA mutations and PFS or OS was analyzed included one study each; therefore, the results of the subgroup analysis were not representative or comparable due to the differences in tumor stage, treatment, and method of ctDNA assessment in the two studies. Melanoma has the highest somatic cell mutation rate [37] and a wide range of genomic alterations. BRAF mutations occur frequently in up to $66 \%$ of patients with melanoma [38], while these activation mutations in BRAF lead to constitutive activation of kinase function independent of upstream signaling from RAS, ultimately promoting cell growth and inhibiting cell apoptosis [39]. NRAS mutations occur in more than $20 \%$ of patients with cutaneous melanoma [40], leading to the activation of MAPK, PI3K, and other cellular signaling pathways, and resulting in cell growth, proliferation, and cell cycle dysfunction. According to the data published by the TCGA network based on whole exome sequence analysis of patients with primary and/or metastatic melanoma, melanoma could be classified into four genomic subtypes: mutant BRAF, mutant RAS, mutant NF1, and Triple-WT (wild-type) [40]. The different genomic subtypes may be of predictive value given the therapeutic targets currently available [41]. Therefore, multigene ctDNA mutation detection can better predict the prognosis of patients with melanoma.

The results of the subgroup analysis classified by method of ctDNA assessment showed that ddPCR was more effective than other methods. The content of ctDNA in peripheral blood is very small, and the content and nucleic acid polymorphisms in different patients vary greatly [42]. Low-sensitivity methods will inevitably miss some of the mutated ctDNA in patients' peripheral blood and produce false negative results. Therefore, the detection of ctDNA requires highly specific and sensitive techniques. DdPCR improves on the advantages of standard PCR, improves accuracy, and reduces error rates [43]. Furthermore, it is cost effective and rapid, allowing highly sensitive detection of allelic variants with a resolution of $0.005 \%$ and simultaneously detecting four different mutations [44]. Although this technique allows the detection of low-frequency mutations, preidentification of target genes is required. The high frequency of BRAF ${ }^{\mathrm{V} 600}$ or NRAS ${ }^{\mathrm{Q} 61}$ mutations in patients 
with melanoma enhances the effectiveness of ddPCR in a majority of the cases [45].

The results of subgroup analysis stratified by sample sources were consistent with the conclusions of Meddeb et al. [46] in that plasma was a better source of ctDNA than serum, this being because, during serum isolation, normal DNA derived from leukocyte lysis during coagulation is much lower than from plasma [47].

There are many biomarkers for the prognosis and prediction of melanoma used clinically. The 8th edition American Joint Committee on Cancer (AJCC) melanoma staging system [48] determined that tumor thickness, mitotic rate, and ulceration were the most dominant prognostic factors. In addition, the only circulating biomarker with significant prognostic value is lactate dehydrogenase (LDH). There are several other circulating biomarkers with diagnostic and prognostic value, such as $\mathrm{C}$-reactive protein (CRP), S100 protein (S100B), melanoma inhibitory activity (MIA), etc. However, the poor sensitivity or specificity of these markers seriously limits their routine use in melanoma [49]. CtDNA is highly fragmented DNA that is distributed by tumor cells into the circulation [50]. A study [51] has shown that ctDNA carries genetic information from the entire tumor genome and is a widely applicable, sensitive, and specific biomarker, thus providing insight into the clonal heterogeneity and evolution of all solid cancers at any time. Our meta-analysis clearly indicated the detection of ctDNA BRAF ${ }^{\mathrm{V} 600}$ and other gene mutations has shown significant value in predicting treatment response and outcome in melanoma. In addition to detecting mutated ctDNA, epigenetic changes and loss of heterozygosity (LOH) of ctDNA can also be detected and analyzed. Two studies by Mori et al. [52, 53] demonstrate the utility of detecting circulating methylated tumor-related genes as a potentially predictive marker of overall survival, and hypermethylation of estrogen receptor $\alpha$ predicting progression-free and overall survival. Due to the small number of relevant studies, we did not conduct a meta-analysis.

In the present meta-analysis, all patients in most of the studies had advanced melanoma, while the patients in one study were at stage II-III. Therefore, our conclusions are applicable to patients with advanced melanoma. Due to the limited number of studies, no subgroup analysis of tumornode-metastasis (TNM) staging was performed. In addition, several limitations in this study should be addressed. We may not have identified published or unpublished studies with negative results, and some studies had a short follow-up time or small sample sizes; therefore, the results should be interpreted with caution. In addition, we hypothesized that possible sources of bias are as follows, and these factors may also lead to heterogeneity: (1) most studies only used univariate analysis, and the size of effect values may be overestimated compared with multivariate analysis; (2) multivariate analysis was used in some studies to obtain accurate estimates, although the confounders used in multivariate analysis were different, which may affect HR values; (3) in most studies, multiple gene ctDNA mutations were detected, which were not identical and may have introduced bias; (4) not all HRs and 95\% CIs included in the meta-analysis were directly collected from the original articles; thus, we extracted survival rates from Kaplan-Meier curves by using Engauge Digitizer version 4.1 [20] to reduce deviation; and (5) follow-up time and cutoff values were not consistent, which may have also caused deviations in the results.

\section{Conclusion}

Despite these limitations, our meta-analysis clearly indicates the prognostic value of ctDNA mutations in melanoma; that is, the presence or elevation of ctDNA mutation or BRAF ${ }^{\mathrm{V} 600} \mathrm{ctDNA}$ mutation was significantly associated with worse prognosis in melanoma patients. However, in view of some of the limitations of this study, the results of our metaanalysis need to be verified by further research. With further exploration and understanding of ctDNA biological function and clinical value, as well as the validation of more large-scale, high-quality prospective studies, ctDNA could eventually be applied in clinical practice and may eventually become part of routine melanoma staging.

\section{Data Availability}

The data supporting this meta-analysis are from previously reported studies, which have been cited. The processed data used to support the findings of this study are included within the article.

\section{Ethical Approval}

All analyses were based on previous published studies; thus, no ethical approval and patient consent are required.

\section{Conflicts of Interest}

The authors declare that they have no conflicts of interest.

\section{Acknowledgments}

This work was supported by the Scientific Research Foundation of Jilin Province (nos. 20200601010JC and 20190701061GH).

\section{Supplementary Materials}

Table S1. Heterogeneity analyses: subgroup analysis of PFS and OS (baseline detectable vs. undetectable). (Supplementary Materials)

\section{References}

[1] J. Larkin, M. Del Vecchio, P. A. Ascierto et al., "Vemurafenib in patients with BRAF ${ }^{\mathrm{V} 600}$ mutated metastatic melanoma: an open-label, multicentre, safety study," The Lancet Oncology, vol. 15, no. 4, pp. 436-444, 2014.

[2] A. Hauschild, J.-J. Grob, L. V. Demidov et al., "Dabrafenib in BRAF-mutated metastatic melanoma: a multicentre, openlabel, phase 3 randomised controlled trial," The Lancet, vol. 380, no. 9839, pp. 358-365, 2012. 
[3] M. K. Callahan, C. R. Flaherty, and M. A. Postow, "Checkpoint blockade for the treatment of advanced melanoma," Melanoma, vol. 167, pp. 231-250, 2016.

[4] A. Ribas, I. Puzanov, R. Dummer et al., "Pembrolizumab versus investigator-choice chemotherapy for ipilimumabrefractory melanoma (KEYNOTE-002): a randomised, controlled, phase 2 trial," The Lancet Oncology, vol. 16, no. 8, pp. 908-918, 2015.

[5] S. L. Topalian, M. Sznol, D. F. Mcdermott et al., "Survival, durable tumor remission, and long-term safety in patients with advanced melanoma receiving nivolumab," Journal of Clinical Oncology, vol. 32, no. 10, pp. 1020-1030, 2014.

[6] H. Shi, W. Hugo, X. Kong et al., "Acquired resistance and clonal evolution in melanoma during BRAF inhibitor therapy," Cancer Discovery, vol. 4, no. 1, pp. 80-93, 2014.

[7] S. J. O'day, M. Maio, V. Chiarion-Sileni et al., "Efficacy and safety of ipilimumab monotherapy in patients with pretreated advanced melanoma: a multicenter single-arm phase II study," Annals of Oncology, vol. 21, no. 8, pp. 1712-1717, 2010.

[8] D. Madhavan, M. Wallwiener, K. Bents et al., "Plasma DNA integrity as a biomarker for primary and metastatic breast cancer and potential marker for early diagnosis," Breast Cancer Research and Treatment, vol. 146, no. 1, pp. 163-174, 2014.

[9] K. N. Maxwell, D. Soucier-Ernst, E. Tahirovic et al., "Comparative clinical utility of tumor genomic testing and cell-free DNA in metastatic breast cancer," Breast Cancer Research and Treatment, vol. 164, no. 3, pp. 627-638, 2017.

[10] E. Takai, Y. Totoki, H. Nakamura et al., "Clinical utility of circulating tumor DNA for molecular assessment in pancreatic cancer," Scientific Report, vol. 5, p. 18425, 2015.

[11] M. K. Kim, S. M. Woo, B. Park et al., "Prognostic implications of multiplex detection of KRAS mutations in cell-free DNA from patients with pancreatic ductal adenocarcinoma," Clinical Chemistry, vol. 64, no. 4, pp. 726-734, 2018.

[12] E. S. Gray, H. Rizos, A. L. Reid et al., "Circulating tumor DNA to monitor treatment response and detect acquired resistance in patients with metastatic melanoma," Oncotarget, vol. 6, no. 39, pp. 42008-42018, 2015.

[13] L. Tan, S. Sandhu, R. J. Lee et al., "Prediction and monitoring of relapse in stage III melanoma using circulating tumor DNA," Annals of Oncology, vol. 30, no. 5, pp. 804-814, 2019.

[14] R. J. Lee, G. Gremel, A. Marshall et al., "Circulating tumor DNA predicts survival in patients with resected high-risk stage II/III melanoma," Annals of Oncology, vol. 29, no. 2, pp. 490-496, 2018.

[15] T. Seremet, Y. Jansen, S. Planken et al., "Undetectable circulating tumor DNA (ctDNA) levels correlate with favorable outcome in metastatic melanoma patients treated with antiPD1 therapy," Journal of Translational Medicine, vol. 17, no. 1, p. 303, 2019.

[16] R. B. Forthun, R. Hovland, C. Schuster et al., "ctDNA detected by ddPCR reveals changes in tumour load in metastatic malignant melanoma treated with bevacizumab," Scientific Reports, vol. 9, no. 1, p. 17471, 2019.

[17] R. E. Board, G. Ellison, M. C. M. Orr et al., "Detection of BRAF mutations in the tumour and serum of patients enrolled in the AZD6244 (ARRY-142886) advanced melanoma phase II study," British Journal of Cancer, vol. 101, no. 10, pp. 1724-1730, 2009.

[18] M. S. Lawrence, P. Stojanov, P. Polak et al., "Mutational heterogeneity in cancer and the search for new cancer-associated genes," Nature, vol. 499, no. 7457, pp. 214-218, 2013.

[19] A. Stang, "Critical evaluation of the Newcastle-Ottawa scale for the assessment of the quality of nonrandomized studies in meta-analyses," European Journal of Epidemiology, vol. 25, no. 9, pp. 603-605, 2010.

[20] M. K. B. Parmar, V. Torri, and L. Stewart, "Extracting summary statistics to perform meta-analyses of the published literature for survival endpoints," Statistics in Medicine, vol. 17, no. 24, pp. 2815-2834, 1998.

[21] J. P. T. Higgins and S. G. Thompson, "Quantifying heterogeneity in a meta-analysis," Statistics in Medicine, vol. 21, no. 11, pp. 1539-1558, 2002.

[22] K. Kozak, A. Kowalik, A. Gos et al., "Cell-free DNA BRAF V600E measurements during BRAF inhibitor therapy of metastatic melanoma: long-term analysis," Tumori, vol. 106, Article ID 300891619900928, 2020.

[23] R. Váraljai, K. Wistuba-Hamprecht, T. Seremet et al., "Application of circulating cell-free tumor DNA profiles for therapeutic monitoring and outcome prediction in genetically heterogeneous metastatic melanoma," JCO Precision Oncology, vol. 3, 2019.

[24] R. Salemi, L. Falzone, G. Madonna et al., "MMP-9 as a candidate marker of response to BRAF inhibitors in melanoma patients with BRAF(V600E) mutation detected in circulating-free DNA," Frontiers in Pharmacology, vol. 9, p. $856,2018$.

[25] A. C. Mcevoy, L. Warburton, Z. Al-Ogaili et al., "Correlation between circulating tumour DNA and metabolic tumour burden in metastatic melanoma patients," BMC Cancer, vol. 18, no. 1, p. 726, 2018.

[26] W. Lu, L. Burton, J. Larkin et al., "Elevated levels of BRAFV600 mutant circulating tumor DNA and circulating hepatocyte growth factor are associated with poor prognosis in patients with metastatic melanoma," JCO Precision Oncology, vol. 2, 2018.

[27] G. Herbreteau, A. Vallée, A.-C. Knol et al., "Quantitative monitoring of circulating tumor DNA predicts response of cutaneous metastatic melanoma to anti-PD1 immunotherapy," Oncotarget, vol. 9, no. 38, pp. 25265-25276, 2018.

[28] M. Gonzalez-Cao, C. Mayo De Las Casas, N. Jordana Ariza et al., "Early evolution of BRAF ${ }^{\mathrm{V} 600}$ status in the blood of melanoma patients correlates with clinical outcome and identifies patients refractory to therapy," Melanoma Research, vol. 28, no. 3, pp. 195-203, 2018.

[29] J. H. Lee, G. V. Long, S. Boyd et al., "Circulating tumour DNA predicts response to anti-PD1 antibodies in metastatic melanoma," Annals of Oncology, vol. 28, no. 5, pp. 1130-1136, 2017.

[30] A. Santiago-Walker, R. Gagnon, J. Mazumdar et al., "Correlation of BRAF mutation status in circulating-free DNA and tumor and association with clinical outcome across four BRAFi and MEKi clinical trials," Clinical Cancer Research, vol. 22, no. 3, pp. 567-574, 2016.

[31] M. Shinozaki, S. J. O'day, M. Kitago et al., "Utility of circulating B-raf DNA mutation in serum for monitoring melanoma patients receiving biochemotherapy," Clinical Cancer Research, vol. 13, no. 7, pp. 2068-2074, 2007.

[32] J. Ferlay, I. Soerjomataram, R. Dikshit et al., "Cancer incidence and mortality worldwide: sources, methods and major patterns in GLOBOCAN 2012," International Journal of Cancer, vol. 136, no. 5, pp. E359-E386, 2015.

[33] Z. Ali, N. Yousaf, and J. Larkin, "Melanoma epidemiology, biology and prognosis," European Journal of Cancer Supplements, vol. 11, no. 2, pp. 81-91, 2013.

[34] F. Spagnolo, V. Picasso, M. Lambertini, V. Ottaviano, B. Dozin, and P. Queirolo, "Survival of patients with metastatic melanoma and brain metastases in the era of MAP-kinase inhibitors and 
immunologic checkpoint blockade antibodies: a systematic review," Cancer Treatment Reviews, vol. 45, pp. 38-45, 2016.

[35] G. Safaee Ardekani, S. M. Jafarnejad, L. Tan, A. Saeedi, and G. Li, "The prognostic value of BRAF mutation in colorectal cancer and melanoma: a systematic review and meta-analysis," PLoS One, vol. 7, no. 10, Article ID e47054, 2012.

[36] P. Mandel and P. Metais, "Nuclear acids in human blood plasma not available," Comptes rendus des séances de la Société de biologie et de ses filiales, vol. 142, no. 3-4, pp. 241-243, 1948.

[37] L. B. Alexandrov, S. Nik-Zainal, D. C. Wedge et al., "Signatures of mutational processes in human cancer," Nature, vol. 500, no. 7463, pp. 415-421, 2013.

[38] H. Davies, G. R. Bignell, C. Cox et al., "Mutations of the BRAF gene in human cancer," Nature, vol. 417, no. 6892, pp. 949-954, 2002.

[39] A. Sharma, N. R. Trivedi, M. A. Zimmerman, D. A. Tuveson, C. D. Smith, and G. P. Robertson, "Mutant V599eb-raf regulates growth and vascular development of malignant melanoma tumors," Cancer Research, vol. 65, no. 6, pp. 2412-2421, 2005.

[40] Cancer Genome Atlas Network, "Genomic classification of cutaneous melanoma,” Cell, vol. 161, no. 7, pp. 1681-1696, 2015.

[41] E. J. Davis, D. B. Johnson, J. A. Sosman, and S. Chandra, "Melanoma: what do all the mutations mean'?" Cancer, vol. 124, no. 17, pp. 3490-3499, 2018.

[42] M. Murtaza, S.-J. Dawson, D. W. Y. Tsui et al., "Non-invasive analysis of acquired resistance to cancer therapy by sequencing of plasma DNA," Nature, vol. 497, no. 7447, pp. 108-112, 2013.

[43] B. Vogelstein and K. W. Kinzler, "Digital PCR," in Proceedings of the National Academy of Sciencesvol. 96, no. 16, pp. 9236-9241, 1999.

[44] V. Taly, D. Pekin, L. Benhaim et al., "Multiplex picodroplet digital PCR to detect KRAS mutations in circulating DNA from the plasma of colorectal cancer patients," Clinical Chemistry, vol. 59, no. 12, pp. 1722-1731, 2013.

[45] L. Calapre, L. Warburton, M. Millward, M. Ziman, and E. S. Gray, "Circulating tumour DNA (ctDNA) as a liquid biopsy for melanoma," Cancer Letters, vol. 404, pp. 62-69, 2017.

[46] R. Meddeb, E. Pisareva, and A. R. Thierry, "Guidelines for the preanalytical conditions for analyzing circulating cell-free DNA," Clinical Chemistry, vol. 65, no. 5, pp. 623-633, 2019.

[47] A. R. Thierry, F. Mouliere, C. Gongora et al., "Origin and quantification of circulating DNA in mice with human colorectal cancer xenografts," Nucleic Acids Research, vol. 38, no. 18, pp. 6159-6175, 2010.

[48] C. M. Balch, J. E. Gershenwald, S.-J. Soong et al., "Final version of 2009 AJCC melanoma staging and classification," Journal of Clinical Oncology, vol. 27, no. 36, pp. 6199-6206, 2009.

[49] P. Vereecken, F. Cornelis, N. Van Baren, V. Vandersleyen, and J.-F. Baurain, "A synopsis of serum biomarkers in cutaneous melanoma patients," Dermatology Research and Practice, vol. 2012, Article ID 260643, 7 pages, 2012.

[50] F. Cheng, L. Su, and C. Qian, "Circulating tumor DNA: a promising biomarker in the liquid biopsy of cancer," Oncotarget, vol. 7, no. 30, pp. 48832-48841, 2016.

[51] C. Bettegowda, M. Sausen, R. J. Leary et al., "Detection of circulating tumor DNA in early- and late-stage human malignancies," Science Translational Medicine, vol. 6, no. 224, Article ID 224ra224, 2014.
[52] T. Mori, S. J. O’Day, N. Umetani et al., "Predictive utility of circulating methylated DNA in serum of melanoma patients receiving biochemotherapy," Journal of Clinical Oncology, vol. 23, no. 36, pp. 9351-9358, 2005.

[53] T. Mori, S. R. Martinez, S. J. O'day et al., "Estrogen receptor- $\alpha$ methylation predicts melanoma progression," Cancer Research, vol. 66, no. 13, pp. 6692-6698, 2006. 